\title{
Cystic fibrosis sputum induces a secretory response from airway gland serous cells that can be prevented by neutrophil protease inhibitors
}

\author{
A. Schuster*, J.V. Fahy*, I. Ueki*, J.A. Nadel**
}

Cystic fibrosis sputum induces a secretory response from airway gland serous cells that can be prevented by neutrophil protease inhibitors. A. Schuster, J.V. Fahy, I. Ueki, J.A. Nadel. @ERS Journals Ltd 1995.

ABSTRACT: High activities of the neutrophil proteases, elastase and cathepsin $G$, are found in the sputum of patients with cystic fibrosis (CF). Because both proteases have been shown to be potent secretagogues for airway submucosal glands, and because hypersecretion is a characteristic feature of $\mathrm{CF}$, the objective of the present study was to examine whether there is secretagogue activity in CF sputum, and to determine the contribution of neutrophil proteases to the secretagogue activity.

Confluent monolayers of cultured bovine tracheal serous cells were pulse-labelled with $\mathrm{Na}_{2}{ }^{35} \mathrm{SO}_{4}$, incubated with diluted $\mathrm{CF}$ sputum supernatants in the presence or absence of different protease inhibitors, and the subsequent release of the radiolabelled macromolecules was measured.

CF sputum potently induced secretion concentration-dependently. Addition of the selective neutrophil elastase inhibitor ICI 200,355 inhibited the secretory response to CF sputum supernatant by $89 \%$. Addition of a cathepsin G-inhibitor resulted in further inhibition of the secretory response. Addition of phosphoramidon, a drug known to inhibit Pseudomonas aeruginosa elastase, had no effect.

We conclude that CF sputum potently stimulates airway submucosal gland cell secretion. These studies with protease inhibitors suggest that neutrophil proteases account substantially for the secretagogue activity present in CF sputum.

Eur Respir J., 1995; 8, 10-14.

Large numbers of neutrophils and high activities of the neutrophil proteases, elastase and cathepsin G, are found in the sputum of patients with cystic fibrosis (CF) [1-4]. Besides the known damaging effects of neutrophil proteases on tissues in CF lungs, both neutrophil elastase and cathepsin $G$ have been shown to be potent secretagogues for cultured bovine tracheal submucosal gland cells [5], and for airway tissue in various species including humans [6]. In fact, neutrophil elastase and cathepsin $G$ have a much greater secretagogue activity in submucosal glands than other known "classic" secretagogues (e.g. methacholine, histamine, etc.) [5, 6]. In humans, airway secretions are believed to originate predominantly from airway gland cells. In CF, a disease characterized by gland hypertrophy and hyperplasia [7], secretagogues of gland cells are likely to be important in the pathophysiology of hypersecretion.

It is our hypothesis that the stimulatory effect of neutrophil proteases on airway gland secretion may play a significant role in the airway hypersecretory state in CF. To investigate this hypothesis, we designed the present study to determine whether CF sputum contains secretagogue activity, and to what extent this activity is due to neutrophil proteases.
*Cardiovascular Research Institute and **Dept of Medicine, University of California, San Francisco, CA, USA.

Correspondence: J.A. Nadel

Cardiovascular Research Institute

Box 0130

University of California

San Francisco

CA 94143 - 0130

USA

Keywords: Airway inflammation airway submucosal gland

hypersecretion

neutrophil elastase

Pseudomonas elastase

Received: March 281994

Accepted after revision September 291994

The study was supported in part by a Cystic Fibrosis Foundation Research Development Program Grant and by HL-24136.

\section{Subjects and sputum samples}

Nine adult CF patients with sputum cultures positive for Pseudomonas aeruginosa were studied during hospitalization for intravenous antimicrobial therapy for exacerbations of pulmonary disease. During hospitalization, daily sputum samples were collected; a total of 18 sputum samples were used in the present study. To obtain solubilized specimens, fresh sputum samples were diluted 1:3 with phosphate-buffered saline (PBS), agitated for $60 \mathrm{~s}$ on a vortex-mixer, centrifuged for $20 \mathrm{~min}$ at $8,800 \times \mathrm{g}$, and the supernatants were collected.

\section{Study design}

After determination of the activities of the neutrophil proteases elastase and cathepsin $\mathrm{G}$ in the $\mathrm{CF}$ sputum samples, confluent monolayers of cultured bovine tracheal gland cells were incubated with different dilutions of the sputum supernatants $(1: 15$ to $1: 30,000)$, and the 
secretory response was assessed by measuring the release of radiolabelled secretory macromolecules from the cells. To determine the relative contributions of proteases to the secretagogue activity of CF sputum, corresponding experiments were carried out in the presence of different protease inhibitors.

\section{Neutrophil protease activities}

The activity of free neutrophil elastase in the sputum supernatants was determined with a specific chromogenic substrate (methoxysuccinyl-L-alanyl-L-alanyl-Lprolyl-L-valyl- $p$-nitroanilide) (Sigma Chemical Co., St. Louis, MO, USA) as described previously [8]. The activity of free cathepsin $G$ in the sputum supernatants was determined photometrically with the selective chromogenic substrate $\mathrm{N}$-succinyl-alanyl-alanyl-prolyl-phenylalanyl-p-nitroanilide (Sigma Chemical Co., St. Louis, MO, USA) as described previously [8]. Purified human neutrophil elastase and cathepsin G (Elastin Products Co., Pacific, MO, USA), respectively, were used as standard references.

\section{Culture of bovine tracheal gland serous cells}

A line of bovine tracheal gland serous cells was cultured as described previously [9]. These cells maintain characteristics of differentiated serous cells, including electron-dense secretory granules [9], and they incorporate radiolabelled precursors into macromolecules and secrete them in response to secretory stimulation $[5,9]$.

\section{Release of ${ }^{35}$ S-labelled macromolecules}

Confluent monolayers of serous cells cultured in flasks with a surface area of $25 \mathrm{~cm}^{2}$ were incubated with $4 \mathrm{ml}$ medium containing $7.5 \mu \mathrm{Ci} \cdot \mathrm{ml}^{-1} \mathrm{Na}_{2}{ }^{35} \mathrm{SO}_{4}$ (ICN Radiochemicals, Inc., Irvine, CA, USA). After 24 h, the medium containing the radiolabel was removed, the cells were washed with PBS, and serum- and antibioticfree medium was added to the flasks; the medium was renewed every $30 \mathrm{~min}$ for $210 \mathrm{~min}$. At $210 \mathrm{~min}$, the medium was collected and either replaced with fresh medium alone (baseline control), or medium containing diluted CF sputum supernatants.

For inhibition studies, diluted sputum supernatants were preincubated with protease inhibitors for $30 \mathrm{~min}$ before addition to the cells; protease inhibitors used were chymostatin, soybean trypsin inhibitor, phosphoramidon (all purchased from Sigma Chemical Co., St. Louis, MO, USA), and the selective neutrophil elastase inhibitor ICI 200,355, [4-(4-bromophenylsulphonylcarbamoyl)benzoyl-L-valyl-L-proline 1 (RS)-(1-trifluoroacetyl-2-methylprolyl)amide] (a gift from ICI Americas, Wilmington, DE, USA) [10, 11].

At $240 \mathrm{~min}$, the medium was collected again, and the spent medium from the 180-210 $\mathrm{min}$ and the 210$240 \mathrm{~min}$ incubation periods was dialysed exhaustively
(Spectra/Por tubing, Spectrum Medical Industries, Inc., Los Angeles, CA, USA; molecular mass cut-off 12,000-14,000 Da) against distilled water to remove unincorporated radiolabelled sulphate. Nondialysable ${ }^{35} \mathrm{~S}$-labelled macromolecules were counted after addition of scintillation fluid (ReadySafe; Beckman Instruments, Inc., Fullerton, CA, USA) by scintillation spectroscopy to an accuracy of 2\% (beta-counter model LS7500; Beckman Instruments, Inc., Irvine, CA, USA). Secretion is expressed as percentage increase of release of ${ }^{35} \mathrm{~S}$ labelled macromolecules during incubation with the diluted CF sputum supernatants over the release during the immediately preceding time period. The calculation was corrected for the slightly declining baseline, which was determined in controls incubated with medium alone.

\section{Analysis of cytotoxicity}

After a $30 \mathrm{~min}$ baseline period, unlabelled serous cells were incubated for 30 min with CF sputum (at the most concentrated dilutions used, 1:30 and 1:15) or with medium containing 1\% Triton-X-100 (Sigma Chemical Co., St. Louis, MO, USA) as a positive control $(n=4$ flasks each), and the release of lactic acid dehydrogenase (LDH) from the cells into the medium was measured spectrophotometrically using a commercially available kit (Sigma Chemical Co., St. Louis, MO, USA). Furthermore, to verify the integrity and viability of the serous cell monolayers after exposure to the diluted sputum supernatants, the culture plates were examined using a phase microscope (IM Zeiss, Zeiss, West Germany) after addition of trypan blue.

\section{Statistical analysis}

All values are expressed as mean \pm sem. Statistical analysis was performed using the paired t-test and the unpaired t-test, as applicable [12]. Significance was attributed to probability values of less than 0.05 .

\section{Results}

Concentrations of neutrophil proteases in CF sputum samples

Mean concentration of catalytically active neutrophil elastase in the sputum samples $(n=18)$ was $180.5 \pm 31.1$ $\mu \mathrm{g} \cdot \mathrm{ml}^{-1}$ (range $34.2-411.7 \mu \mathrm{g} \cdot \mathrm{ml}^{-1} ;=1.3 \times 10^{-6}-1.5 \times 10^{-5} \mathrm{M}$ ). Mean concentration of catalytically active cathepsin $\mathrm{G}$ was $29.7 \pm 6.1 \mu \mathrm{g} \cdot \mathrm{ml}^{-1}$ (range $4.9-62.3 \mu \mathrm{g} \cdot \mathrm{ml}^{-1} ;=1.6 \times 10^{-7}-$ $2.1 \times 10^{-6} \mathrm{M}$ ). Effect of diluted CF sputum on secretion of sulphated
macromolecules

Cultured serous cells secreted nondialysable ${ }^{35} \mathrm{~S}$-labelled macromolecules spontaneously and continuously at a low 


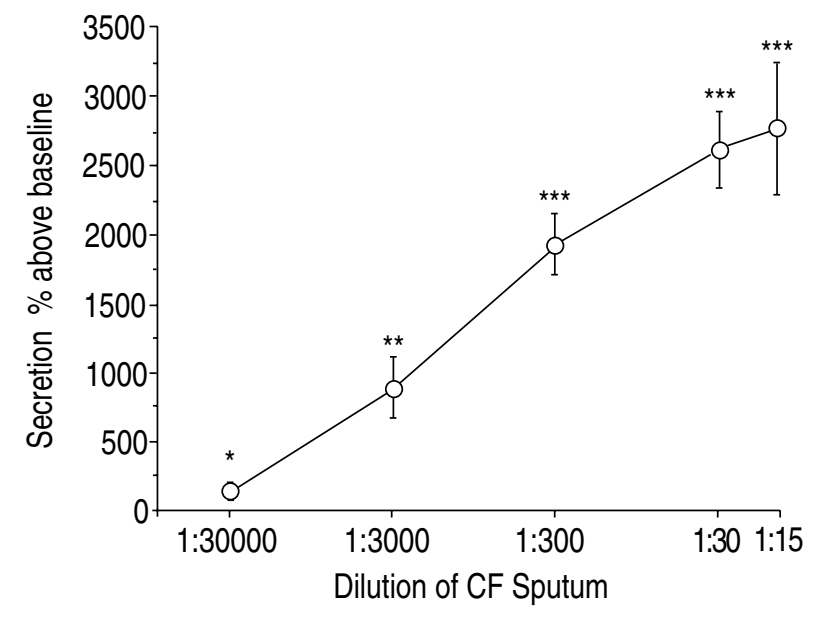

Fig. 1. - Effect of incremental dilutions of sputum from patients with cystic fibrosis $(\mathrm{CF})(\mathrm{n}=9$ for $1: 30,000$ to $1: 30 ; n=6$ for $1: 15)$ on secretion of ${ }^{35} \mathrm{~S}$-labelled macromolecules from cultured tracheal gland serous cells. Secretion is markedly stimulated, being significantly differrent from baseline at a 1:30,000 dilution, and reaching a maximum at a $1: 30$ dilution. Values are means \pm SEM. ${ }^{*}: \mathrm{p}<0.05 ;{ }^{* *}: \mathrm{p}<0.001$; ${ }^{* * *}: \mathrm{p}<0.0001$ versus baseline secretion.

rate. $C F$ sputum $(n \geq 6)$ markedly stimulated secretion (fig. 1): a significant increase in secretion over baseline $(141 \pm 60 \%)$ was measured at a dilution of $1: 30,000$ $(\mathrm{p}<0.05)$, and with less dilution the secretory response increased.

Secretion induced by diluted CF sputum was not cytotoxic. At the highest concentrations studied, incubation with CF sputum did not cause a significant increase of LDH release above baseline $\left(15.1 \pm 5.1 \mathrm{U} \cdot \mathrm{ml}^{-1}\right.$ $\mathrm{LDH}$ baseline versus $18.9 \pm 6.2 \mathrm{U} \cdot \mathrm{ml}^{-1} \mathrm{LDH}$ after incubation with the $1: 30$ dilution; $13.2 \pm 6.3$ versus $15.7 \pm 4.2 \mathrm{U} \cdot \mathrm{ml}^{-1} \mathrm{LDH}$ for the $1: 15$ dilution), while incubation with $1 \%$ Triton-X-100 caused a 15 fold increase in LDH $\left(8.8 \pm 3.0\right.$ versus $\left.133.3 \pm 6.7 \mathrm{U} \cdot \mathrm{ml}^{-1} \mathrm{LDH}\right)$. As examination by phase microscopy showed, cell monolayers after exposure to 1:30 and 1:15 diluted sputum remained intact, and the cells excluded trypan blue.

Inhibition of secretion induced by CF sputum diluted to a defined neutrophil elastase concentration

In the first set of inhibition experiments ( $n=5$ each), supernatants from sputum samples were diluted to a final concentration of $10^{-8} \mathrm{M}$ neutrophil elastase. The secretory response from cultured serous cells was $2,048 \pm 195 \%$ above baseline. Addition of the selective inhibitor of neutrophil elastase, ICI 200,355 inhibited this secretory response in a dose-dependent fashion (fig. 2 ). At $10^{-6} \mathrm{M}$, ICI 200,355 inhibited the secretory response to diluted $\mathrm{CF}$ sputum supernatants by $88.7 \pm 3.5 \%$. The simultaneous addition of the cathepsin $\mathrm{G}$ inhibitor chymostatin to ICI 200,355 resulted in a further, significant decrease in the secretory responses to sputum supernatants compared to ICI 200,355 alone $(\mathrm{p}<0.05)$; in the presence of ICI $200,355\left(10^{-6} \mathrm{M}\right)$ plus chymostatin (50 $\left.\mu \mathrm{g} \cdot \mathrm{ml}^{-1}\right)$, there was $96.9 \pm 1.3 \%$ inhibition of the secretory response. A comparable result was obtained when we used ICI $200,355\left(10^{-6} \mathrm{M}\right)$ plus soybean trypsin

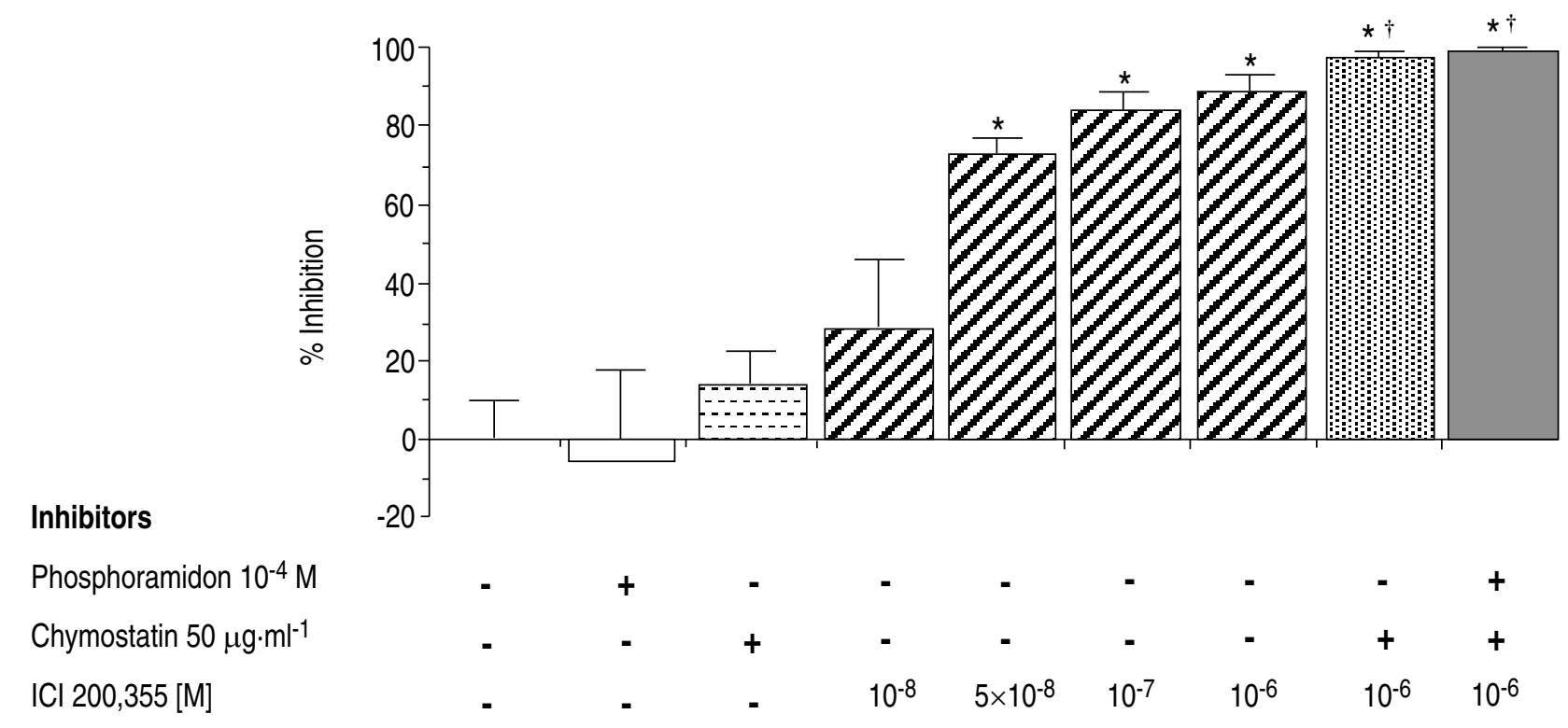

Fig. 2. - Effect of protease inhibitors on secretion of ${ }^{35} \mathrm{~S}$-labelled macromolecules from cultured tracheal glands serous cells induced by cystic fibrosis (CF) sputum supernatants diluted to a final concentration of $10^{-8} \mathrm{M}$ neutrophil elastase (NE). Values are means \pm SEM, $\mathrm{n}=5$ for each inhibition experiment. The Pseudomonas elastase inhibitor, phosphoramidon alone, $(\square)$ has no effect on the secretory response. The cathepsin $\mathrm{G}$ inhibitor, chymostatin, alone $(--)$ ) has a small inhibitory effect, but it does not reach significance. The selective neutrophil elastase inhibitor, ICI 200,355, ( 2 ) inhibits the secretory response in a concentration-dependent fashion. Further addition of the cathepsin G inhibitor, chymostatin, (E: in almost complete inhibition. Thus, the further addition of the Pseudomonas elastase inhibitor, phosphoramidon, ( $\square$ ) does not change the secretory response further. $\quad$ : secretion (\% above baseline) is significantly different from secretion induced by CF sputum diluted to $10^{-8} \mathrm{M}$ NE $(\mathrm{p}<0.002)$; $\dagger$ : secretion (\% above baseline) is significantly different from sputum-induced secretion in the presence of ICI 200,355 (10-6 M) alone $(\mathrm{p}<0.05)$. 


\section{Inhibitors}

Phosphoramidon $10^{-4} \mathrm{M}$
Chymostatin $50 \mu \mathrm{g} \cdot \mathrm{ml}^{-1}$
$\mathrm{ICl} 200,35510^{-5} \mathrm{M}$

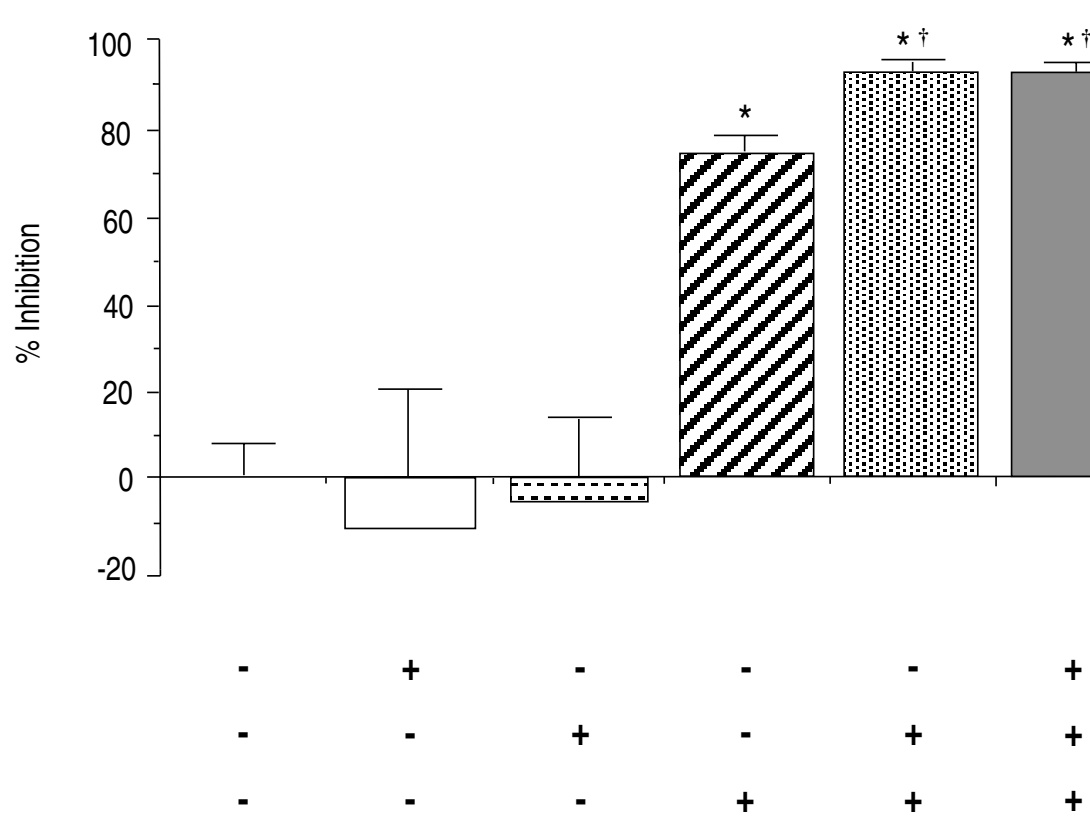

Fig. 3. - Effect of protease inhibitors on secretion of ${ }^{35} \mathrm{~S}$-labelled macromolecules from cultured tracheal glands serous cells induced by 1:30 diluted cystic fibrosis (CF) sputum. Values are means \pm SEM, $n=5$ for each inhibition experiment. The Pseudomonas elastase inhibitor, phosphoramidon, alone ( $\square$ ) and the cathepsin $\mathrm{G}$ inhibitor, chymostatin, alone ( $-\sigma$ ) have no effect on the secretory response. The selective neutrophil elastase inhibitor, ICI 200,355 ( $)$, markedly inhibits the secretory reponse to diluted CF sputum supernatants, and addition of chymostatin ( in almost complete inhibition. Thus, the further addition of phosphoramidon $(\square)$ does not change the secretory response further. $\star$ : secretion (\% above baseline) is significantly different from secretion induced by CF sputum diluted 1:30 (p<0.0002); †:secretion $(\%$ above baseline) is significantly different from sputum-induced secretion in the presence of ICI $200,355\left(10^{-5} \mathrm{M}\right)$ alone $(\mathrm{p}<0.01)$.

inhibitor $\left(100 \mu \mathrm{g} \cdot \mathrm{ml}^{-1}\right)$ to inhibit neutrophil elastase and cathepsin $\mathrm{G}$ in the sputum supernatants $(97.5 \pm 0.7 \%$ inhibition). When phosphoramidon $\left(10^{-4} \mathrm{M}\right)$, an inhibitor of Pseudomonas aeruginosa elastase, was added together with both neutrophil protease inhibitors, there was no further change in the secretory response. Also, phosphoramidon alone $\left(10^{-4} \mathrm{M}\right)$ had no mean inhibitory effect on sputum supernatant-induced secretion.

\section{Inhibition of secretion induced by 1:30 diluted CF sputum samples}

In the second set of inhibition experiments ( $\mathrm{n}=5$ each), we studied CF sputum samples all diluted by the same factor, 1:30. There was a secretory response from cultured serous gland cells of 2,647 $\pm 213 \%$ above baseline in these experiments. At this relatively high concentration of CF sputum supernatant, addition of the cathepsin $\mathrm{G}$ inhibitor chymostatin $\left(50 \mu \mathrm{g} \cdot \mathrm{ml}^{-1}\right)$ or of the Pseudomonas elastase inhibitor phosphoramidon $\left(10^{-4} \mathrm{M}\right)$ alone did not affect the secretory response (fig. 3). In the presence of the neutrophil elastase inhibitor ICI $200,355\left(10^{-5} \mathrm{M}\right)$, the secretory response was markedly reduced by $74.8 \pm 3.9 \%(\mathrm{p}<0.0002)$. Simultaneous addition of chymostatin $\left(50 \mu \mathrm{g} \cdot \mathrm{ml}^{-1}\right)$ to ICI $200,355\left(10^{-5} \mathrm{M}\right)$, inhibited the secretory response even further by $92.7 \pm$ $2.8 \%(\mathrm{p}<0.005$ compared to inhibition by ICI 200,355 alone). Further addition of phosphoramidon $\left(10^{-4} \mathrm{M}\right)$ did not change the secretory response further (92.8 \pm $1.8 \%$ inhibition).

\section{Discussion}

Hypersecretion is a characteristic feature of airway disease in $\mathrm{CF}$, and the major cause of death is the progressive deterioration of respiratory function as a consequence of chronic accumulation of secretions associated with infection and airway inflammation [13]. Because sputum from CF patients contains high concentrations of neutrophil proteases, and because neutrophil proteases are the most potent secretagogues for airway secretory cells yet described $[5,6]$, we hypothesized that these proteases might play an important role in the airway hypersecretion that is so important clinically in this disease. At the same time, we recognized that other mediators which have been demonstrated to exist in $\mathrm{CF}$ sputum, such as prostaglandins, leukotrienes and the products of Pseudomonas bacteria (e.g. Pseudomonas elastase), are known to be secretagogues [10, 14, 15] and might also play significant roles.

To test our hypothesis, a method of study was required. We reasoned that airway luminal material might provide a reasonable reflection of secretagogue activity present in airway tissue, and we decided to examine sputum. The availability of a permanent line of airway submucosal gland cells that retain secretory capability and that can be used as a bioassay system for secretagogues greatly facilitated the study [9].

Firstly, we found that sputum from patients with $\mathrm{CF}$ induced profound secretagogue activity in the test system, even when diluted 30,000 fold, and we showed that this effect was not due to cytotoxicity. Then, we tested the hypothesis that neutrophil proteases are important 
secretagogues in CF sputum by examining the effects of selective protease inhibitors on gland cell secretion. We found that most of the secretagogue effect of the sputum was prevented by pretreatment with a selective inhibitor of neutrophil elastase. The remainder of the secretagogue effect was prevented by administration of an inhibitor of cathepsin G; depending on the experimental conditions, the contribution of cathepsin $\mathrm{G}$ varied between $8-18 \%$. After pretreatment with a combination of neutrophil elastase and cathepsin $G$ inhibitors, virtually all secretagogue activity of the sputum was abolished. These findings indicate that the secretagogue activity present in CF sputum is virtually entirely explicable on the basis of neutrophil proteases.

All of the patients studied had chronic Pseudomonas aeruginosa infections, and Pseudomonas elastase is a potent secretagogue for airway gland cells [10]. Nevertheless, phosphoramidon, an inhibitor of Pseudomonas elastase, had no effect on sputum-induced secretion in the present studies. This may be due to inactivation of Pseudomonas elastase in vivo by specific antibodies to the enzyme. Evidence to support this hypothesis derives from the fact that more than $98 \%$ of sputum elastolytic activity in CF sputum is reported to be due to neutrophil elastase alone [2]. From our results, we conclude that bacterial elastase is not an important source of secretagogue activity in the patient group studied. Nor do other mediators present in CF sputum, such as arachidonate metabolites seem to play a significant role as secretagogues in CF airways; this might possibly be due to proteolytic degradation and inactivation of these substances.

In summary, CF sputum causes a striking secretory response in airway gland cells in culture, and this effect is virtually completely blocked by inhibitors of neutrophil proteases. Although these sputum studies suggest a role for neutrophil protease inhibitors in the treatment of the hypersecretory state of the airways in $\mathrm{CF}$, studies evaluating the efficacy of this form of therapy in patients will be required to finally determine the importance of neutrophil proteases in the pathogenesis of hypersecretion and airway obstruction in this disease.

Acknowledgements: The authors thank C.B. Basbaum, and W.E. Finkbeiner, for supplying cultured bovine tracheal serous cells. They are grateful to M. Stulbarg, who assisted with the referral of patients. They thank $\mathrm{H}$. Wong and P. Massion, for helping to collect and process daily sputum samples. They also thank N. Holt for the preparation of the manuscript. A.S. is the recipient of a fellowship from the Deutsche Forschungsgemeinschaft. J.F. is the recipient of a Travelling Studentship in Medicine from the National University of Ireland.

\section{References}

1. Pennington JE, Wolff SM, Puziss M. Summary of a workshop on infections in patients with cystic fibrosis. J Infect Dis 1979; 140: 252-256.

2. Tournier J-M, Jacquot J, Puchelle E, Bieth JG. Evidence that Pseudomonas elastase does not inactivate the bronchial inhibitor in the presence of leukocyte elastase. Am Rev Respir Dis 1985; 132: 524-528.

3. Goldstein W, Döring G. Lysosomal enzymes from polymorphonuclear leukocytes and proteinase inhibitors in patients with cystic fibrosis. Am Rev Respir Dis 1986; 134: 49-56.

4. Suter S, Schaad UB, Tegner H, Ohlsson K, Desgrand champs D, Waldvogel FA. Levels of free granulocyte elastase in bronchial secretions from patients with cystic fibrosis: effect of antimicrobial treatment against Pseudomonas aeruginosa. J Infect Dis 1986; 153: 902909.

5. Sommerhoff CP, Nadel JA, Basbaum CB, Caughey GH. Neutrophil elastase and cathepsin G stimulate secretion from cultured bovine airway gland serous cells. J Clin Invest 1990b; 85: 682-689.

6. Schuster A, Ueki I, Nadel JA. Neutrophil elastase stimulates tracheal submucosal gland secretion that is inhibited by ICI 200,355. Am J Physiol (Lung Cell Mol Physiol) 1992; 262: L86-L91.

7. Sobonya RE, Taussig LM. Quantitative aspects of lung pathology in cystic fibrosis. Am Rev Respir Dis 1986; 134: 290-295.

8. Fahy JV, Schuster AS, Ueki I, Boushey HA, Nadel JA. Mucus hypersecretion in bronchiectasis: the role of neutrophil proteases. Am Rev Respir Dis 1992; 146: 1430-1433.

9. Finkbeiner WE, Nadel JA, Basbaum CB. Establishment and characterization of a cell line derived from bovine tracheal glands. In Vitro 1986; 22: 561-567.

10. Sommerhoff CP, Krell, RD, Williams JL, Gomes BC, Strimpler AM, Nadel JA. Inhibition of human neutrophil elastase by ICI 200,355. Eur J Pharmacol 1991; 193: 153-158.

11. Williams JC, Falcone RC, Knee C, et al. Biologic characterization of ICI 200,880 and ICI 200,355, novel inhibitors of human neutrophil elastase. Am Rev Respir Dis 1991; 144: 875-883.

12. Zar JH. In: Biostatistical Analysis. 2nd edn. Englewood Cliffs, NJ, Prentice-Hall, 1984; pp. 120-160.

13. Tizzano EF, Buchwald M. Cystic fibrosis: beyond the gene to therapy. J Pediatr 1992; 120: 337-349.

14. Zakrzewski JT, Barnes NC, Costello JF, Piper PJ. Lipid mediators in cystic fibrosis and chronic obstructive pulmonary disease. Am Rev Respir Dis 1987; 136: 779-782.

15. Lundgren JD, Shelhamer JH. Pathogenesis of airway mucus hypersecretion. J Allergy Clin Immunol 1990; 85: 399-417. 